\title{
A fotoantropometria como método de análise facial para estimativa de idade forense:
}

\section{revisão sistemática}

\section{Photoanthropometry as a method of facial analysis for forensic age estimation: a systematic review}

Fotoantropometría como método de análisis facial para estimación de la edad forense: revisión sistemática

Yanka Barbosa Alves

ORCID: https://orcid.org/0000-0001-7653-2263 Universidade Federal da Paraíba, Brasil

E-mail: yankajp@gmail.com

Tainá Nascimento Falcão

ORCID: https://orcid.org/0000-0002-3326-063X Universidade Federal da Paraíba, Brasil

E-mail: taina.falcao@hotmail.com

Loyse Martorano Fernandes

ORCID: https://orcid.org/0000-0002-5995-5085

Universidade Estadual de Campinas, Brasil

E-mail: loyse martorano@hotmail.com

Johnys Berton Medeiros da Nóbrega

ORCID: https://orcid.org/ 0000-0002-9009-5044 Universidade Federal da Paraíba, Brasil

E-mail: johnysberton@gmail.com

Laíse Nascimento Correia Lima

ORCID: https://orcid.org/0000-0002-1773-847X Universidade Federal da Paraíba, Brasil E-mail: laiselima@msn.com

Carlos Eduardo Palhares Machado

ORCID: https://orcid.org/0000-0002-0306-6211 Polícia Federal, Brasil

E-mail: palhares.cepm@dpf.gov.br

Bianca Marques Santiago

ORCID: https://orcid.org/0000-0001-9559-913X Universidade Federal da Paraíba, Brasil E-mail: bianca.santiago@yahoo.com.br

\section{Resumo}

O crescimento exponencial de crimes na internet, como a pedofilia, demanda muitas vezes a estimativa da idade a partir das imagens faciais dos indivíduos envolvidos. O objetivo desse estudo foi avaliar a aplicabilidade do método da fotoantropometria para estimativa de idade com fins forenses por meio de uma revisão sistemática. Realizaram-se buscas nas bases PUBMED, SCOPUS, The Cochrane Library, Web of Science, LILACS, CAPES e SIGLE, utilizando MeSH e termos livres com base na estratégia PICO (P: população; I: intervenção; C: comparação; e O: desfecho). Incluíram-se estudos que avaliaram imagens de indivíduos por fotoantropometria para estimativa de idade forense. Procedeu-se extração dos dados e avaliação da qualidade metodológica (QUADAS-2). De 2809 artigos identificados, 37 foram selecionados inicialmente por abordar análise facial através de fotoantropometria. Após leitura na íntegra, identificou-se que 9 estudos que utilizaram o método para estimativa de idade. Com amostra proveniente de 9 nacionalidades diferentes, 5 artigos tiveram suas marcações fotoantropométricas realizadas manualmente, enquanto 4 foram automatizadas. Todos os estudos tiveram agrupamentos de idade próprios para determinação das mesmas, não havendo coincidências entre eles. O método da fotoantropometria foi considerado eficaz para estimativa de idade em 5 artigos, enquanto 4 reportam que mais estudos são necessários para minimizar erros. Somente 3 estudos apresentaram baixo risco de viés por utilizarem padrão de referência aplicável e confiável. A fotoantropometria apresenta aplicabilidade limitada na estimativa de idade forense, havendo evidência questionável acerca de seu uso, devido aos riscos de viés presentes nos estudos.

Palavras-chave: Revisão sistemática; Odontologia legal; Grupos etários; Face; Fotografia. 
Research, Society and Development, v. 10, n. 2, e60010212469, 2021

(CC BY 4.0) | ISSN 2525-3409 | DOI: http://dx.doi.org/10.33448/rsd-v10i2.12469

\begin{abstract}
The exponential growth of Internet crime, such as pedophilia, often requires estimating age from the facial images of the individuals involved. The objective of this study was to evaluate the applicability of the photoanthropometric method for estimating age with forensic purposes by means of a systematic review. We searched the databases PUBMED, SCOPUS, The Cochrane Library, Web of Science, LILACS, CAPES and SIGLE, using MeSH and free terms based on PICO strategy (P: population, I: intervention, C: comparison, and O: outcome). We included studies that evaluated images of individuals by photoanthropology to estimate forensic age. Data extraction and methodological quality evaluation were performed (QUADAS-2). Of 2809 articles identified, 37 were initially selected for addressing facial analysis through photoanthropology. After reading in full, 9 studies that used the method for age estimation remained. With a sample from 9 different nationalities, 5 articles had their photoanthropometric markings done manually, while 4 were automated. All studies had age groupings for their determination, and there were no coincidences between them. The method of photoanthropometry was considered effective for age estimation in 5 articles, while 4 reported that more studies are needed to minimize errors. Only 3 studies had low risk of bias because they used an applicable and reliable reference standard. Photoanthropometry has limited applicability in the forensic age estimation, and there is questionable evidence that supports its use due to the risks of bias present in the studies.
\end{abstract}

Keywords: Review; Forensic dentistry; Age groups; Face; Photography.

\title{
Resumen
}

El crecimiento exponencial de delitos en Internet, como la pedofilia, requiere, frecuentemente, una estimación de edad a partir de imágenes faciales de las personas involucradas. El objetivo fue evaluar la aplicabilidad del método de fotoantropometría, para estimar la edad con fines forenses, mediante una revisión sistemática. Las búsquedas se materializaron en las bases de datos PUBMED, SCOPUS, The Cochrane Library, Web of Science, LILACS, CAPES y SIGLE, utilizando MeSH y términos de búsqueda, basados en la estrategia PICO (P: población; I: intervención; C: comparación; y O: resultado). Fueron incluidos estudios que evaluaron imágenes de individuos por fotoantropometría, para estimar la edad forense. Se realizó extracción de datos y evaluación de la calidad metodológica (QUADAS-2). De 2809 artículos identificados, se seleccionaron, inicialmente 37, para abordar el análisis facial por medio de fotoantropometría. Luego de la lectura en su totalidad, se constató que 9 estudios usaron dicho método para estimar edad. Con una muestra de 9 nacionalidades diferentes, 5 artículos tuvieron sus marcaciones fotoantropométricas efectuadas manualmente y 4, automatizadas. Todos tenían sus propios grupos de edad, sin coincidencia entre ellos. El método de fotoantropometría resultó efectivo para estimar la edad en 5 artículos, y 4 informaron que se necesitan más estudios para minimizar errores. Solo 3 tuvieron un bajo riesgo de sesgo, al emplear un estándar de referencia aplicable y confiable. La fotoantropometría tiene una aplicabilidad limitada en la estimación de la edad forense, con evidencia cuestionable que no respalda su uso, debido al riesgo de sesgo presente en los estudios.

Palabras clave: Revisión sistemática; Odontología legal; Grupos de edad; Cara; Fotografía.

\section{Introduçãa}

Nos últimos anos, tem sido observado um aumento constante na distribuição de material de pornografia infantil, devido à facilidade de compartilhamento através da web (Kleinhans, 2004). Em consequência disso, a requisição de perícia para avaliação dessas imagens é relativamente comum para realizar a estimativa de idade das pessoas envolvidas.

Em geral, a má qualidade da imagem e a baixa resolução fornecem mais obstáculos para a avaliação da idade dos indivíduos representados nas imagens, em comparação com o exame direto de indivíduos vivos (Bednarek, 2006; Cattaneo et al., 2012; Gehlen et al., 2005).

É notório também a importância do desenvolvimento de métodos adequados para estimar a idade de crianças e adolescentes representados em imagens e/ou fitas pornográficas (Cummaudo et al., 2014).

Estimar a idade de alguém por meio de imagens 2D ainda não é exato por não existir métodos cientificamente válidos (Cattaneo et al., 2012; Cummaudo et al., 2014; FISWG, 2012). Porém, ultimamente a possibilidade de usar medidas faciais para fins forenses tem sido explorada (Cattaneo et al., 2012; FISWG, 2012).

O Grupo de Trabalho Científico de Identificação Facial (FISWG - Facial Identification Scientific Working Group) foi criado com o intuito de desenvolver padrões e melhores práticas nas comparações faciais baseadas em imagens. De acordo com ele, a identificação facial pode ser realizada por quatro métodos: Holísticos, Morfológicos, Fotoantropométricos, e por 
superposição (FISWG, 2012).

A antropometria usada em fotografias, denominada de fotoantropometria, tem vantagens sobre outros métodos antropométricos. Ela é não-invasiva, tem baixo custo, além de ser rápida, reprodutível e as imagens poderem ser arquivadas para futura avaliação (Becker et al., 1998; Driessen et al., 2011; Schaaf et al., 2010; Wall et al., 1999).

Os sistemas digitais estão cada vez mais presentes nas empresas e organizações, apresentando vantagens e desvantagens. Nas vantagens estão inseridas imagens de melhor qualidade e facilidade de uso do sistema. Nas desvantagens, tem-se as exportações das imagens: elas são comprimidas e detalhes cruciais podem ser perdidos. Na tentativa de obter melhores imagens das câmeras de vigilância e eliminar uma possível desvantagem, é necessário ter clareza sobre os objetivos de montar um sistema de vigilância. A instalação de câmeras em determinadas posições depende da intenção, como impedir que os funcionários roubem ou para fornecer segurança a funcionários e clientes de possíveis ofensores (Kleinberg et al., 2007).

Câmeras colocadas em ângulos altos não necessariamente fornecerão os detalhes necessários para identificação; no entanto, câmeras colocadas em ângulos baixos correm o risco de a visão ser bloqueada. O tipo de lente na câmera de vídeo também pode afetar o que é capturado em fita de vídeo (Kleinberg et al., 2007).

Com isso, o presente estudo buscou identificar evidências quanto a aplicabilidade do método da fotoantropometria para análise facial com fins forenses por meio de uma revisão sistemática, cuja pergunta norteadora é: "A fotoantropometria é um método adequado para realizar estimativa de idade com fins forenses?".

\section{Metodologia}

A metodologia que foi desenvolvida está de acordo com o protocolo para revisões sistemáticas, proposto pelo Preferred Reporting Items for Systematic Reviews and Metanalysis (PRISMA) (Liberati et al., 2009; Pereira et al., 2018) e incluiu as etapas: formulação da estratégia de busca da literatura, seleção de estudos a partir de critérios de inclusão e exclusão, seleção de artigos, avaliação da qualidade metodológica e risco de vieses dos estudos selecionados, extração e tabulação de dados.

A busca da literatura foi realizada de maneira independente por dois pesquisadores, que discutiram previamente os critérios de busca, de maneira a selecionar artigos publicados até maio de 2018, independentemente do idioma de publicação.

Primeiramente e tendo como base a estratégia PICO (Maia \& Antonio, 2012), foram definidos três temas que foram combinados pelo operador booleano "AND". Cada tema foi criado pela utilização do operador "OR" e foram pesquisados em palavras do título, resumo e palavras-chave utilizadas na indexação (MeSH terms).

$\mathrm{O}$ primeiro tema foi criado para incluir as diferentes formas de mencionar a população $(\mathrm{P})$ de interesse da pergunta central da presente revisão sistemática, e incluíram as seguintes palavras-chaves: "face" ou "photography"; o segundo para representar a intervenção (I), ou seja, o método de análise facial, incluindo: "face mapping", "facial recognition", "facial comparison", "image processing, compter-assisted", "metric analysis" e "anthropometry"; e o terceiro tema para representar as finalidades forenses (O), incluindo: "forensic sciences", "forensic dentistry", "Forensic Anthropology", "Age determination", "Age estimation", "Sex Determination Analysis", "Ethnic Groups", "Stature determination" e "Stature estimation".

Após registro do protocolo no Prospero (CRD 42018084032), essas sintaxes foram utilizadas para efetuar a busca por artigos de pesquisa originais nas bases de dados: PUBMED, SCOPUS, The Cochrane Library, Web of Science, LILACS, CAPES (no catálogo de dissertações e teses) e System for Information on Grey Literature (SIGLE), tendo sido adaptada para cada uma das bases (Tabela 1). Também foram pesquisadas as referências dos artigos selecionados para identificar os estudos que não foram capturados na pesquisa inicial.

Nos critérios de elegibilidade, foram incluídos os estudos transversais e longitudinais com imagens (fotografia ou vídeo) 
Research, Society and Development, v. 10, n. 2, e60010212469, 2021

(CC BY 4.0) | ISSN 2525-3409 | DOI: http://dx.doi.org/10.33448/rsd-v10i2.12469

de indivíduos que foram avaliadas por meio de análises métricas (método da fotoantropometria) para fins forenses de estimativa de idade. Foram excluídos revisões da literatura, editoriais, relatos de caso, série de casos e estudos analíticos que não apresentassem resultados quanto a aplicabilidade do método para fins forenses.

A avaliação da qualidade metodológica e do risco de vieses dos estudos selecionados foi realizada a partir da utilização do QUADAS 2 (https://www.nice.org.uk/article/pmg6b/chapter/Appendix-F-Methodology-checklist-the-QUADAS-2-tool-forstudies-of-diagnostic-test-accuracy), o qual é recomendado para estudos de acurácia de testes diagnósticos. No entanto, como um método de análise facial não se constitui de um teste de diagnóstico típico, foram necessárias algumas adaptações para adequar algumas questões à técnica, como as propostas por Lopes (2012) para estudos de estimativa de idade com fins forenses.

Tabela 1 - Sintaxe utilizada em cada uma das bases eletrônicas para realização das buscas por artigos.

\title{
BASE DE \\ DADOS
}

\begin{abstract}
Web of Science $\quad$ (TS=(face) OR TS=(faces) OR TS=(photography) OR TS=(photographies)) AND (TS=("face recognition") OR $\mathrm{TS}=($ "face perception") OR TS=(anthropometry) OR TS=("Face mapping") OR TS=("Facial comparison") OR $\mathrm{TS}=$ ("Image processing Computer Assisted") OR TS=("Computer assisted Image processing") OR TS=("Image Reconstruction") OR TS=("Image Reconstructions") OR TS=("Image analysis computer assisted") OR $\mathrm{TS}=($ "Computer assisted image analysis") OR TS=("Computer-assisted image analyses") OR TS=("Metric Analysis")) AND (TS=("forensic sciences") OR TS=("forensic science") OR TS=("Forensic dentistry") OR TS=("Forensic Anthropology") OR TS=("Human identification") OR TS=("Human identifications") OR TS=("Age determination") OR TS=("Age estimation") OR TS=("Sex Determination analysis") OR TS=("Sex Determination Analyses") OR TS=("Sex determination technique") OR TS=("Sex determination techniques") OR TS=("Sex determination technics") OR TS=("Sex determination technic") OR TS=("Ethnic groups") OR TS=("Ethnic groups") OR TS=(nacionality) OR TS=(nacionalities) OR TS=("stature determination") OR TS=("stature estimation"))
\end{abstract}

Medline-

PubMed
$((((()(($ Forensic Dentistry[MeSH Terms]) OR Forensic Dentistry[Title/Abstract]) OR Dentistry Forensic[Title/Abstract]) OR Forensic Odontology[Title/Abstract]) OR Age determination by teeth[MeSH Terms]) OR Age determination by teeth[Title/Abstract] OR Age estimation[Title/Abstract]))) AND ( (()((()(()((()(()(adolescent[MeSH Terms]) OR adolescent[Title/Abstract]) OR Adolescents[Title/Abstract]) OR Adolescence[Title/Abstract]) OR Teen[Title/Abstract]) OR Teens[Title/Abstract]) OR Teenager[Title/Abstract]) OR Teenagers[Title/Abstract]) OR Youth[Title/Abstract]) OR Youths[Title/Abstract]) OR Young adult[MeSH Terms]) OR Young adult[Title/Abstract]) OR Young adult [Title/Abstract]) OR Adulthood[Title/Abstract]) OR Molar, Third[MeSH Terms]) OR Molar Third[Title/Abstract]) OR Molars Third[Title/Abstract]) OR Third Molar[Title/Abstract]) OR Third Molars[Title/Abstract]))
( TITLE-ABS-KEY ( face ) OR TITLE-ABS-KEY ( photography ) OR TITLE-ABS-KEY ( photographies ) ) AND ( TITLE-ABS-KEY ( "face recognition" ) OR TITLE-ABS-KEY ( "face perception" ) OR TITLE-ABSKEY ( anthropometry ) OR TITLE-ABS-KEY ( "Face mapping" ) OR TITLE-ABS-KEY ( "Facial comparison" ) OR TITLE-ABS-KEY ( "Image processing Computer Assisted" ) OR TITLE-ABS-KEY ( "Computer assisted Image processing" ) OR TITLE-ABS-KEY ( "Image Reconstruction" ) OR TITLE-ABS-KEY ( "Image Reconstructions" ) OR TITLE-ABS-KEY ( "Image analysis computer assisted" ) OR TITLE-ABS-KEY ( "Computer assisted image analysis" ) OR TITLE-ABS-KEY ( "Computer-assisted image analyses" ) OR TITLEABS-KEY ( "Metric Analysis" ) ) AND ( TITLE-ABS-KEY ( "forensic sciences" ) OR TITLE-ABS-KEY ( "forensic science" ) OR TITLE-ABS-KEY ( "Forensic dentistry" ) OR TITLE-ABS-KEY ( "Forensic Anthropology" ) OR TITLE-ABS-KEY ( "Human identification" ) OR TITLE-ABS-KEY ( "Human identifications" ) OR TITLE-ABS-KEY ( "Age determination" ) OR TITLE-ABS-KEY ( "Age estimation" ) OR TITLE-ABS-KEY ( "Sex Determination analysis" ) OR TITLE-ABS-KEY ( "Sex Determination Analyses" ) OR TITLE-ABS-KEY ( "Sex determination technique" ) OR TITLE-ABS-KEY ( "Sex determination techniques" ) OR TITLE-ABS-KEY ( "Sex determination technics" ) OR TITLE-ABS-KEY ( "Sex determination technic" ) OR TITLE-ABS-KEY ( "Ethnic groups" ) OR TITLE-ABS-KEY ( nacionality ) OR TITLE-ABS-KEY ( nacionalities ) OR TITLE-ABS-KEY ( "stature determination" ) OR TITLE-ABS-KEY ( "stature estimation" )) 
LILACS

(tw:((mh:(face)) OR (tw:(face)) OR (tw:(cara)) OR (tw:(face)) OR (tw:(faces)) OR (mh:(photography)) OR (tw:( photography)) OR (tw:(fotografía)) OR (tw:(fotografia)) OR (tw:(photographies)) )) AND (tw:((mh:("Facial Recognition")) OR (tw:( "Facial Recognition")) OR (tw:( "Reconocimiento Facial")) OR (tw:( "Reconhecimento Facial”)) OR (tw:(“Face Perception”)) OR (tw:(“Identity Recognition”)) OR (tw:(Adolescencia)) OR (tw:(Adolescência)) OR (mh:(Anthropometry)) OR (tw:( Anthropometry)) OR (tw:(Antropometría)) OR (tw:(Antropometria)) OR (tw:("face mapping")) OR (tw:(“facial comparison")) OR (tw:("metric analysis")) OR (mh:("image processing compter-assisted)) OR (tw:("image processing compter-assisted")) OR (tw:("image processing computer assisted")) OR (tw:("computer assisted image processing")) OR (tw:("image reconstruction")) OR (tw:("image reconstructions")) OR (tw:("computer assisted image analysis")) OR (tw:("computer-assisted image analyses")) )) AND (tw:((mh:(“forensic sciences")) OR (tw:(“forensic sciences”)) OR (tw:(“ciencias foreses")) OR (tw:( "ciências forenses")) OR (tw:("forensic science”)) OR (mh:("forensic dentistry")) OR (tw:("forensic dentistry”)) OR (mh:(“forensic anthropology”)) OR (tw:(“forensic anthropology”)) OR (tw:(“antropología forense”)) OR (tw:(“antropologia forense”)) OR (tw:(“human identification”)) OR (tw:("human identifications")) OR (tw:(“age determination")) OR (tw:(“age estimation")) OR (mh:("sex determination analysis")) OR (tw:("sex determination analysis")) OR (tw:("sex determination analyses")) OR (tw:("sex determination techniques")) OR (tw:("sex determination technique")) OR (tw:("sex determination tecnics")) OR (tw:("sex determination tecnic")) OR (mh:("ethnic groups")) OR (tw:(“ethnic groups")) OR (tw:(“ethnic group”)) OR (tw:(nationality)) OR (tw:(nationalities)) (tw:(“stature determination”)) OR (tw:(“stature estimation”))))

\section{CAPES (Face) AND (“Facial recognition”) AND ("Forensic Sciences”)}

SIGLE

$\begin{array}{lll}\text { COCHRANE } & \# 1 & \text { MeSH descriptor: [Face] explode all trees } \\ \# 2 & \text { Face* } \\ \# 3 & \# 1 \text { or \#2 } \\ \# 4 & \text { MeSH descriptor: [Photography] explode all trees } \\ \# 5 & \text { Photograph* } \\ \# 6 & \# 4 \text { or \#5 } \\ \# 7 & \text { \#3 or \#6 } \\ \# 9 & \text { "FeSH descriptor: [Facial Recognition] explode alltrees } \\ \# 10 & \# 8 \text { or \#9 } \\ \# 11 & \text { MeSH descriptor: [Anthropometry] explode all trees } \\ \# 12 & \text { Anthropometry } \\ \# 13 & \# 11 \text { or \#12 } \\ \# 14 & \# 10 \text { or \#13 } \\ \# 15 & \text { MeSH descriptor: [Image Processing, Computer-Assisted] explode all trees } \\ \# 16 & \text { "Image processing computer assisted" or "computer assisted image processing" or "image reconstruction*" } \\ \text { or "image analysis computer assisted" or "computer assisted image analysis" or "computer-assisted image analyses" } \\ \# 17 & \text { \#15 or \#16 } \\ \# 18 & \text { \#14 or \#17 } \\ \# 19 & \text { "Face mapping" or "Facial comparison" or "metric analysis" } \\ \# 20 & \text { \#18 or \#19 }\end{array}$

(Face OR Faces OR Photography OR Photographies) AND ("Facial recognition" OR "Face perception" OR Anthropometry OR "Face mapping" OR "Facial comparison" OR "Metric analysis" OR "Image processing computer assisted" OR "Image processing computed assisted" OR "Computer assisted Image processing" OR "Image reconstrution" OR "Image reconstrutions" OR "Image analysis computer assisted" OR "Computer assisted image analysis" OR "Computer-assisted image analyses" OR "Image analyses Computer-assisted") AND ("Forensic Sciences" OR "Forensic Science" OR"Forensic Dentistry" OR "Forensic Anthropology" OR "Human identification" OR "Human identifications" OR "Age Determination" OR "Age estimation" OR "Sex determination Analysis" OR "Sex Determination Analyses" OR "Sex Determination Techniques" OR "Sex Determination Technique" OR "Sex Determination Technic" OR "Sex Determination Technics" OR "Ethnic groups" OR "Ethnic group" OR Nationality OR Nationalities OR "Stature Determination" OR "Stature Estimation”) 
Research, Society and Development, v. 10, n. 2, e60010212469, 2021

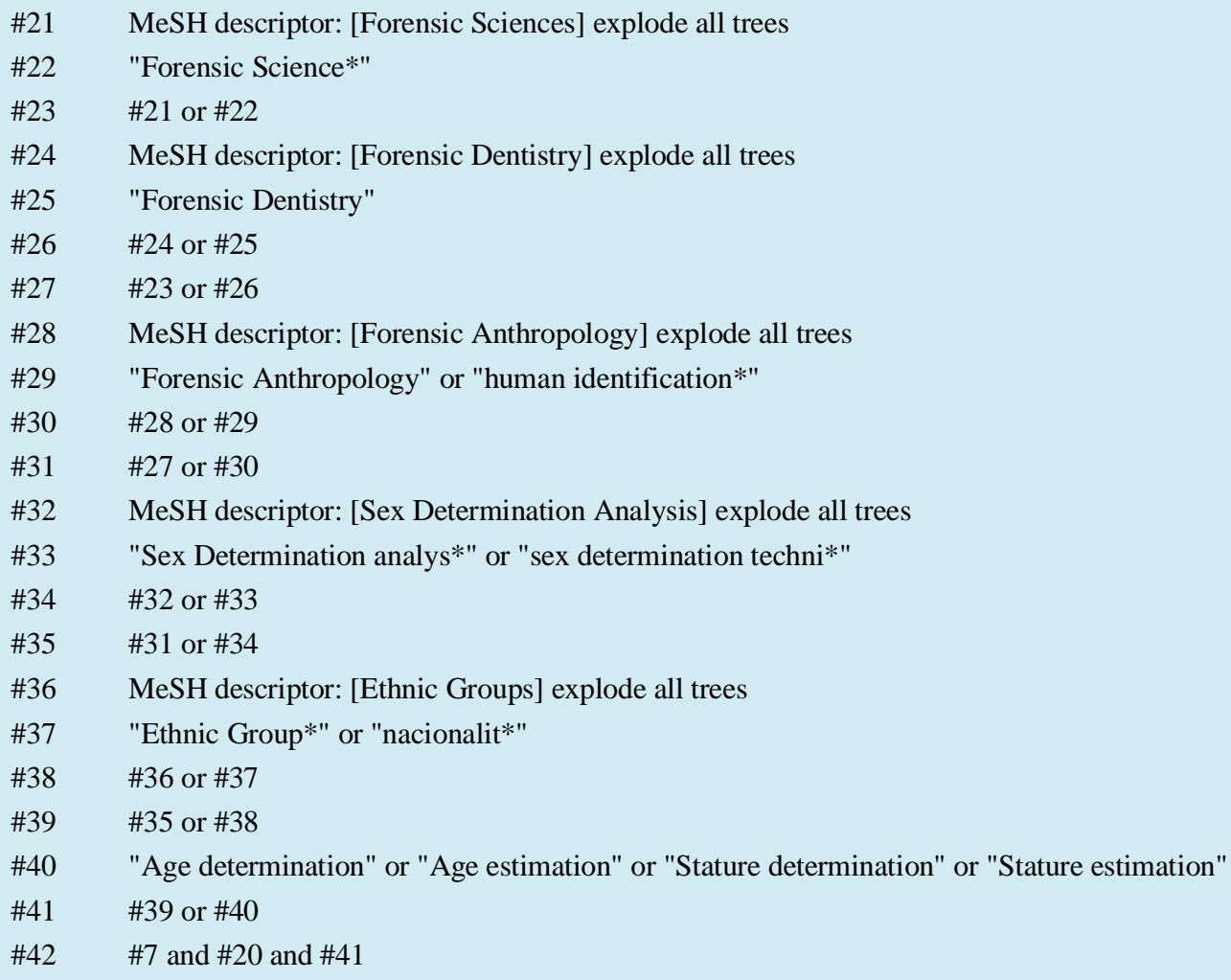

Fonte: Dados do próprio estudo.

\section{Resultados}

Foram identificados 2809 registros de todas as bases de dados selecionadas até a fase da leitura na íntegra, que através da ferramenta Mendeley Desktop, foram importados para remoção das duplicadas e leitura por título e abstract. Após esta fase 37 estudos foram selecionados inicialmente e, em seguida, após ter acesso aos estudos em formato completo e leitura na íntegra dos mesmos, permaneceu o total de 09 artigos (Figura 1 e Quadro 1). 
Research, Society and Development, v. 10, n. 2, e60010212469, 2021

(CC BY 4.0) | ISSN 2525-3409 | DOI: http://dx.doi.org/10.33448/rsd-v10i2.12469

Figura 1 - Diagrama PRISMA - Fluxograma das etapas desenvolvidas para a seleção de estudos primários que respondessem a pergunta foco da revisão sistemática.

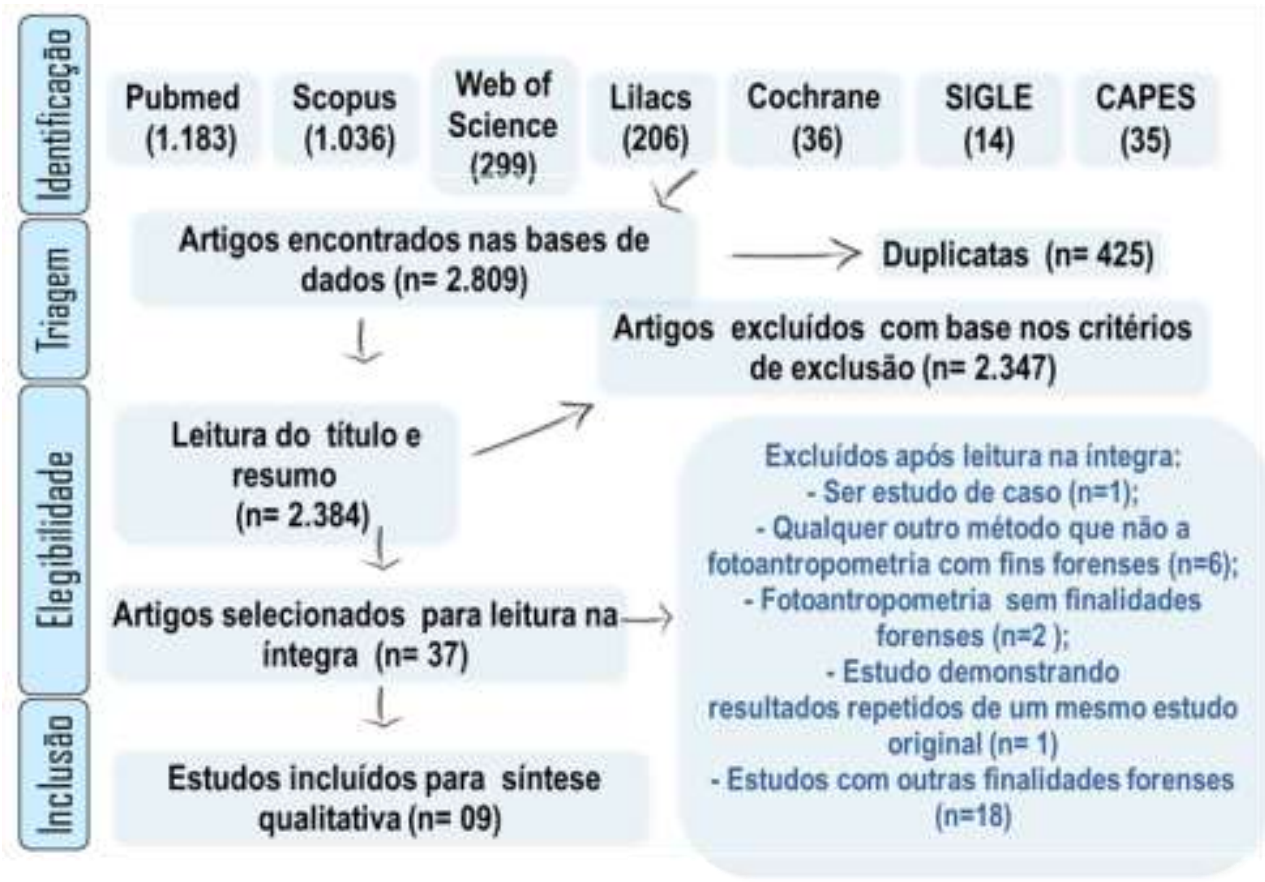

Fonte: Dados do próprio estudo. 
Quadro 1 - Estudos incluídos nessa revisão sistemática e a revista onde foi publicada.

\begin{tabular}{|c|c|c|c|}
\hline Ano & Autores & Título & Revista \\
\hline 2007 & Meijerman et. al. & Cross-Sectional Anthropometric Study of theExternal Ear & J Forensic Sci \\
\hline 2012 & Cattaneo et. al. & $\begin{array}{l}\text { Can facial proportions taken from images be of use for ageing in cases of } \\
\text { suspected child pornography? A pilot study }\end{array}$ & Int J Legal Med \\
\hline 2012 & Jahanbin et. al. & $\begin{array}{l}\text { Evaluation of Some Facial Anthropometric Parameters inan Iranian Population: } \\
\qquad \text { Infancy Through Adolescence }\end{array}$ & J Craniofac Surg \\
\hline 2014 & Cummaudo et. al. & $\begin{array}{l}\text { Towards a method for determining age ranges from faces of juveniles on } \\
\text { photographs }\end{array}$ & Forensic Sci Int \\
\hline 2014 & Ratnayake et. al. & $\begin{array}{l}\text { The juvenile face as a suitable age indicator in childpornography cases: a pilot } \\
\text { study on the reliability of automated and visual estimation approaches }\end{array}$ & Int J Legal Med \\
\hline 2015 & Imaizumi et. al. & $\begin{array}{l}\text { Three-dimensional analyses of aging-induced alterations in facialshape: a } \\
\text { longitudinal study of } 171 \text { Japanese males }\end{array}$ & Int J Legal Med \\
\hline 2016 & Farrera et. al. & Quantitative assessment of the facial features of a Mexican populationdataset & Forensic Sci Int \\
\hline 2017 & Santosh-Kumar et. al. & $\begin{array}{l}\text { An Active Age Estimation of Facial image using Anthropometric Model and } \\
\text { Fast ICA }\end{array}$ & J Eng Sci Tec Rev \\
\hline 2017 & Machado et. al. & A new approach for the analysis of facialgrowth and age estimation: Iris ratio & PLOS ONE \\
\hline
\end{tabular}

Fonte: Dados do próprio estudo.

Após essa etapa, os dados dos estudos incluídos foram extraídos para montagem da Tabela de Extração de Dados (Documento Suplementar - Apêndice 1), onde verifica-se abrangência de 09 nacionalidades (População da Itália, Lituânia, Alemanha, México, Japão, Irã, Brasil, Holanda e Índia); com agrupamentos de idade próprios para determinação das mesmas.

De acordo com o detalhamento desses estudos, ainda podem-se observar algumas diferenças, como: 6 artigos com marcações realizadas manualmente enquanto 3 são automatizados; 8 estudos fazem uso da face completa, enquanto só 1 utiliza dimensões verticais da orelha; e a respeito do padrão utilizado, 4 artigos fizeram uso da norma frontal e lateral, outros 4 utilizaram apenas a normal frontal e 1 estudo só a norma lateral.

Um achado dessa revisão é que o método da fotoantropometria foi considerado um método adequado para finalidade forense de estimativa de idade em 05 artigos, enquanto 04 estudos consideraram que mais estudos são necessários para minimizar erros.

A qualidade metodológica analisada pelo QUADAS-2 (Tabela 2) indicou que apenas 3 dos 9 trabalhos foram considerados com baixo risco de viés por utilizarem padrão de referência aplicável e confiável. Alto risco de viés foi atribuído a 4 estudos sobre o domínio referente a seleção de pacientes, o que é explicada pela utilização de determinada população do local 
Research, Society and Development, v. 10, n. 2, e60010212469, 2021

(CC BY 4.0) | ISSN 2525-3409 | DOI: http://dx.doi.org/10.33448/rsd-v10i2.12469

de origem do estudo.

Ainda sobre o QUADAS-2, na Figura 2 observam-se as proporções de dos estudos, sendo classificados em baixo risco, alto risco e risco não claro quanto ao risco de viés e preocupação com a aplicabilidade, que é gerado pelo próprio domínio. Verifica-se bastante representatividade de estudos com alto risco (67\%; $n=6)$ no que diz respeito ao viés dos estudos. Quanto a aplicabilidade do estudo, $35 \%(n=3)$ dos artigos receberam a classificação de "risco não claro" por não conter informações sobre a referência padrão a ser utilizada, onde o ideal seria ter como padrão as medições “in vivo" para ser comparadas às medições nas fotografias.

Tabela 2 - Resultados da qualidade metodológica usando o QUADAS-2.

\begin{tabular}{|c|c|c|c|c|c|c|c|}
\hline \multirow[t]{2}{*}{ ESTUDOS } & \multicolumn{4}{|c|}{ RISCO DE VIÉS } & \multicolumn{3}{|c|}{$\begin{array}{c}\text { PREOCUPAÇÕES DE } \\
\text { APLICABILIDADE }\end{array}$} \\
\hline & $\begin{array}{l}\text { Seleção de } \\
\text { Pacientes }\end{array}$ & $\begin{array}{c}\text { Índice do } \\
\text { teste }\end{array}$ & $\begin{array}{c}\text { Referência } \\
\text { Padrão }\end{array}$ & $\begin{array}{c}\text { Fluxo e } \\
\text { temporalidade }\end{array}$ & $\begin{array}{l}\text { Seleção de } \\
\text { Pacientes }\end{array}$ & $\begin{array}{c}\text { Índice do } \\
\text { Teste }\end{array}$ & $\begin{array}{c}\text { Referência } \\
\text { Padrão }\end{array}$ \\
\hline
\end{tabular}

\section{Cattaneo et.}

al. (2012)

(:)

()

(-)

Cummaudo

et. al.

6

(2)

(2)

()

()

(2014)

Farrera et.

al. (2016)

6

()

()

(2)

Imaizumi

et. al.

6

(2)

(2015)

Jahanbin et

al. (2012)

6

()

()

(-)

()

(2)

(2)

Machado et.

al. (2017)

(2)

()

()

Meijerman

et. al.

(2007)

6

()

?

(-)

?

Ratnayake

et. al.

(2014)

(2)

$?$

(-)

()

Santosh-

Kumar et.

al. (2017)

()

(2)

(i) 
Figura 2 - Gerado a partir dos resultados da Qualidade Metodológica QUADAS-2

$\square$ Baixo

Proporção dos estudos sendo baixo, alto ou não claro quanto a APLICABILIDADE

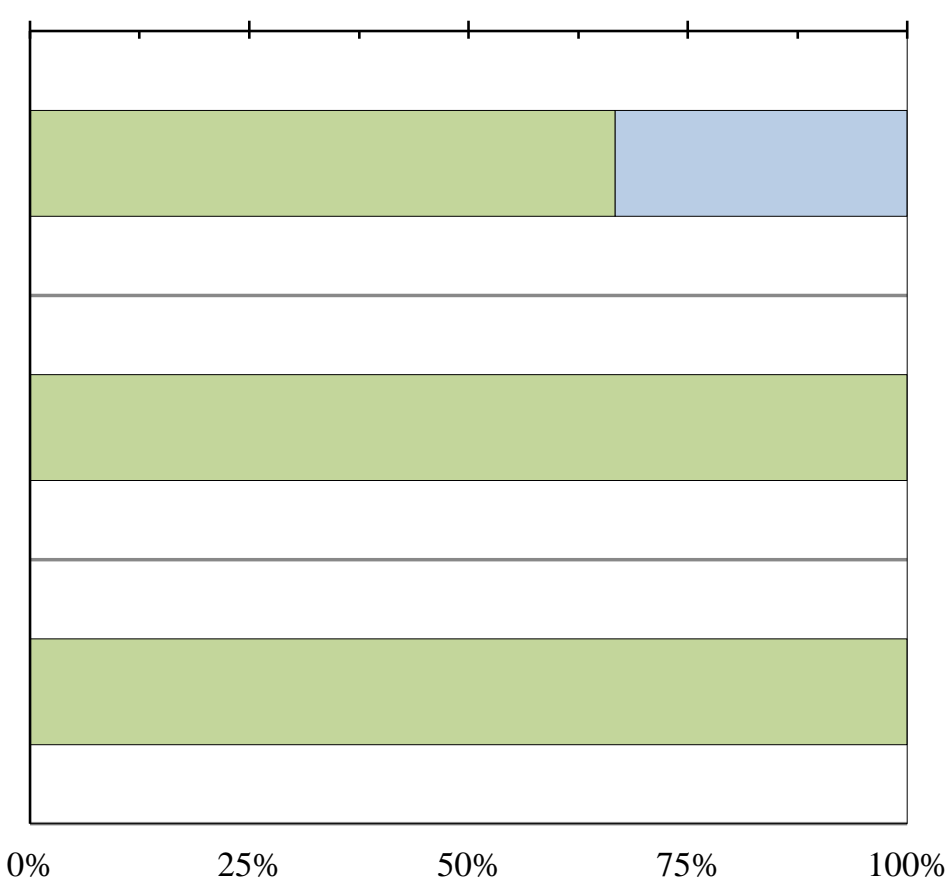

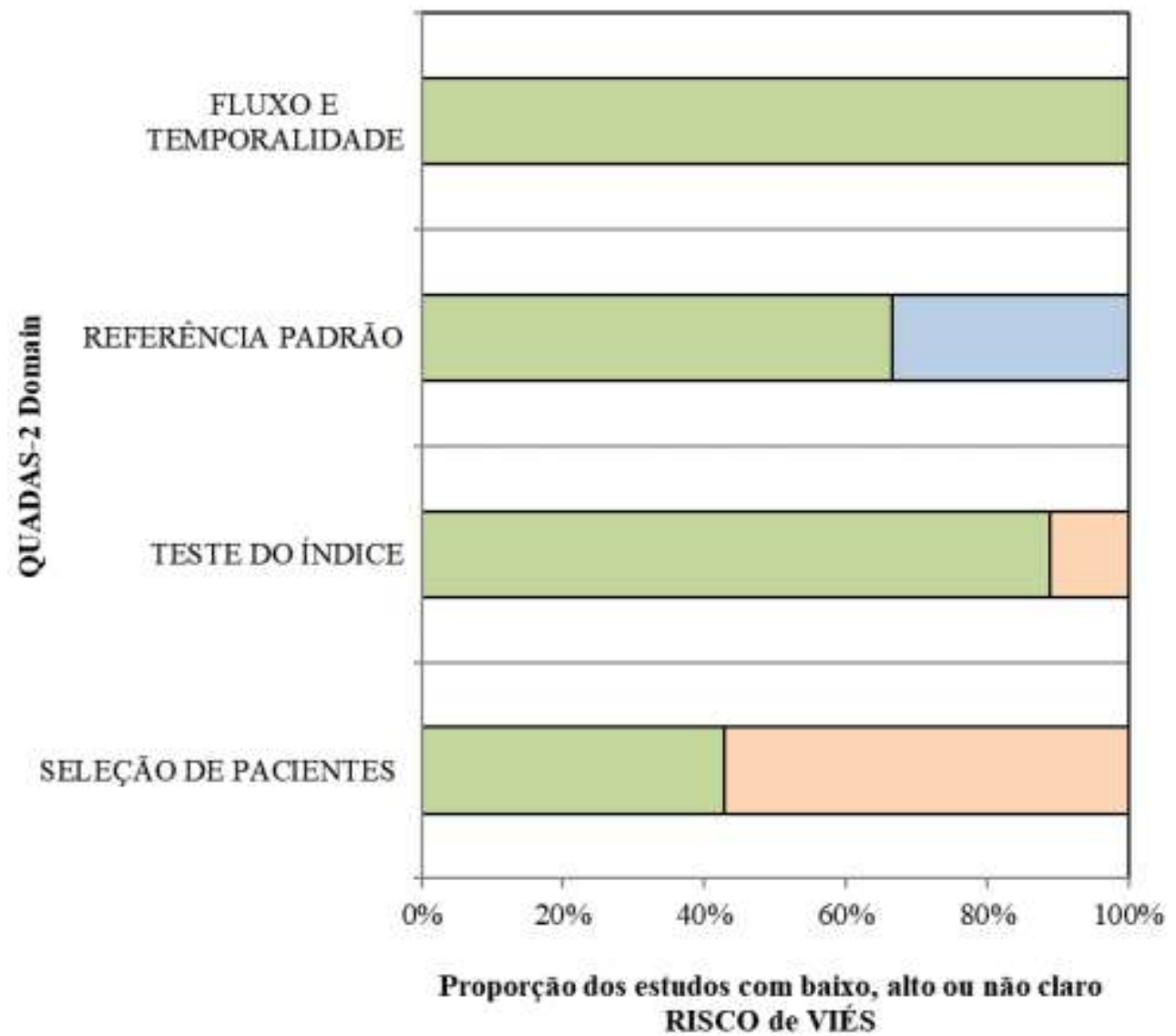

RISCO de VIÉS

Fonte: Dados do próprio estudo. 


\section{Discussão}

A literatura (Akdeniz, 2016; Borges et al., 2018) aponta que a internet é uma rede de globalização que traz muitos modos de uso, e um deles é quando se compartilha material ilegal, incluindo a pornografia infantil. Trata-se de um grande problema atual, que demanda constante esforço de muitos países e instituições para prevenir e combater, por meio da abordagem dos infratores.

A estimativa de idade realizada pela fotoantropometria é algo pretendido como um método seguro para ser utilizado em material pedo-pornográfico (Cattaneo et al., 2012). Tal abordagem também pode ser útil na verificação da idade dos rostos de menores em fotografias em casos de crianças desaparecidas e/ou casos de identificação de crianças (Cummaudo et al., 2014).

Segundo alguns estudos (Borges et al., 2018; Cattaneo et al., 2009, 2012), em fotos de pessoas nuas, algumas faixas etárias (principalmente sendo igual ou menor de 18 anos) são elementares para legalmente serem reconhecidos como crimes de pornografia. Contudo, há um envolvimento da população preocupada com essa questão, e apesar disso, tem sido difícil ter uma resolutividade satisfatória pela maioria dos métodos que geralmente são aplicados.

Ser capaz de prever a forma facial de um indivíduo em diferentes idades tem muitas aplicações práticas. E uma delas vai ao encontro com a estimativa de idade, por exemplo, a análise da imagem de uma criança sequestrada, em que os especialistas podem estimar, isto é, fazer uma projeção de como seria sua forma facial depois de muitos anos em caso de desaparecidos (Jahanbin et al., 2013; Ratnayake et al., 2014).

Um dos estudos (Jahanbin et al., 2013) incluídos nessa revisão que se mostrou com baixo risco de viés e alta aplicabilidade mostra que a análise visual da face leva a estimativa da idade razoáveis dentro dos limites da variabilidade biológica natural.

Os resultados dos artigos de Cummaudo et al. (2014), Davis et al. (2010), Galvanék et. al. (2015), e Introna et. al. (2007), mostram que ao abordar os estudos antropométricos em imagens 2D, é de fundamental importância a avaliação da confiabilidade do posicionamento dos pontos de referência faciais, a fim de evitar erros grosseiros e confirmar a importância dos marcos biológicos na avaliação facial. Há a necessidade de mais trabalhos serem desenvolvidos para se certificar da confiabilidade desses achados em uma amostra representativa, incluindo mais categorias etárias(Cattaneo et al., 2012).

Para que a fotoantropometria seja utilizada nos tribunais de forma aceitável e confiável, Moreton e Morley (2011) retratam que é essencial que a influência das variáveis extrínsecas sejam investigadas experimentalmente, com o intuito de obter vários indicativos de confiabilidade do resultado obtido.

No que diz respeito a estimativa de idade, alguns estudos (Driessen et al., 2011; Machado et al., 2017; Schmeling et al., 2008) expressam que são necessários parâmetros padronizados para orientar e melhorar as práticas forenses em nível internacional. Isso traz também uma forma de poder se utilizar mais da fotoantropometria e cada vez mais aperfeiçoá-la, a fim de trazer uma segurança nos resultados.

O risco de viés quanto a população foi considerado baixo nos estudos de Cattaneo et. al. (2012) e Ratnayake et. al. (2014) pelos motivos de utilizarem na sua amostra fotografias de mais de uma nacionalidade envolvida. Isso é explicado pelo método poder ser utilizado em qualquer lugar do mundo, trazendo referências aos diversos padrões faciais dos países, onde cada região contém traços faciais característicos comuns e nítidos entre si.

Foi considerado baixo risco de viés quanto à população também o estudo de Machado et. al. (Machado et al., 2017), que se utiliza de pessoas da região sudeste do país, devido ao fato da população brasileira ser muito miscigenada, não apresentando um padrão facial que não poderia ser aplicado a outros países.

Os artigos (Davis et al., 2010; Kleinberg et al., 2007) reportam situações nas quais os métodos são agrupados a fim de obter um resultado em comum. Apresentar-se convincente, comprobatório e conduzir veracidade é o que é buscado por esses estudos a qualquer intuito de resposta sobre questionamentos com fins forenses. 
É considerado benéfico para a ciência a utilização de vários métodos para se equivaler os resultados, contribuindo como incentivo para os pesquisadores continuarem buscando a temática com mais evidências científicas. Contudo, tem-se mais eficácia do método da fotoantropometria agrupado a outros métodos a fim de trazer veracidade e comprovação dos fatos.

\section{Conclusão}

Conclui-se que a fotoantropometria apresenta aplicabilidade limitada na estimativa de idade forense, havendo evidência questionável acerca de seu uso, devido aos riscos de viés presentes nos estudos. É importante a complementação de estudos primários com a fotoantropometria em busca de uma metodologia aplicável a fim de padronizar o método em busca de eficácia em todos os padrões faciais que são distintos em diferentes nacionalidades.

\section{Agradecimentos}

Ao Conselho Nacional de Desenvolvimento Científico e Tecnológico (CNPq) e a Pró-reitora de Pesquisa (PROPESQ) da Universidade Federal da Paraíba (UFPB) pelo incentivo à produção científica, financiando por meio de bolsa do Programa Institucional de Bolsas de Iniciação Científica (PIBIC) - vigência 2017/2018.

\section{Referências}

Akdeniz, Y. (2016). Internet child pornography and the law: National and international responses. Routledge.

Becker, M., Svensson, H., \& Jacobsson, S. (1998). Clinical examination compared with morphometry of digital photographs for evaluation of repaired cleft lips. Scandinavian journal of plastic and reconstructive surgery and hand surgery, 32(3), 301-306.

Bednarek, J. (2006). [Problems associated with chronological age estimation of children exploited in child pornography production]. Archiwum medycyny sadowej i kryminologii, 56(3), 149-154.

Borges, D. L., Vidal, F. B., Flores, M. R. P., Melani, R. F. H., Guimaraes, M. A., \& Machado, C. E. P. (2018). Photoanthropometric face iridial proportions for age estimation: An investigation using features selected via a joint mutual information criterion. Forensic Science International, 284, 9-14. https://doi.org/10.1016/j.forsciint.2017.12.011

Cattaneo, C., Obertova, Z., Ratnayake, M., Marasciuolo, L., Tutkuviene, J., Poppa, P., Gibelli, D., Gabriel, P., \& Ritz-Timme, S. (2012). Can facial proportions taken from images be of use for ageing in cases of suspected child pornography? A pilot study. International Journal of Legal Medicine, 126(1), 139-144. https://doi.org/10.1007/s00414-011-0564-7

Cattaneo, C., Ritz-Timme, S., Gabriel, P., Gibelli, D., Giudici, E., Poppa, P., Nohrden, D., Assmann, S., Schmitt, R., \& Grandi, M. (2009). The difficult issue of age assessment on pedo-pornographic material. Forensic science international, 183(1-3), e21-e24.

Cummaudo, M., Guerzoni, M., Gibelli, D., Cigada, A., Obertova, Z., Ratnayake, M., Poppa, P., Gabriel, P., Ritz-Timme, S., \& Cattaneo, C. (2014). Towards a method for determining age ranges from faces of juveniles on photographs. Forensic Science International, 239 , 107.e1-7. https://doi.org/10.1016/j.forsciint.2014.01.021

Davis, J. P., Valentine, T., \& Davis, R. E. (2010). Computer assisted photo-anthropometric analyses of full-face and profile facial images. Forensic science international, 200(1-3), 165-176.

Driessen, J. P., Vuyk, H., \& Borgstein, J. (2011). New insights into facial anthropometry in digital photographs using iris dependent calibration. International journal of pediatric otorhinolaryngology, 75(4), 579-584.

FISWG. (2012). Guidelines for facial comparison methods. Facial Identification Scientific Working Group.

Galvánek, M., Furmanová, K., Chalás, I., \& Sochor, J. (2015). Automated facial landmark detection, comparison and visualization. Proceedings of the 31st spring conference on computer graphics, 7-14.

Gehlen, S., Broker, H. M., Ritz-Timme, S., Tuktuviene, J., \& Cattaneo, C. (2005). Child pornography: Development of a method for identification of faces as childish. Second International Conference on Reconstruction of Soft Facial Parts, RheinAhrCampus Remagen.

Introna, F., De Donno, A., Santoro, V., \& Carbonara, M. (2007). Poor reliability of facial indices for comparative metric facial identification without parametrical superimposition. Forensic Science, Medicine, and Pathology, 3(4), 275-282.

Jahanbin, A., Rashed, R., Yazdani, R., Shahri, N. M., \& Kianifar, H. (2013). Evaluation of some facial anthropome tric parameters in an Iranian population: Infancy through adolescence. Journal of Craniofacial Surgery, 24(3), 941-945.

Kleinberg, K. F., Vanezis, P., \& Burton, A. M. (2007). Failure of anthropometry as a facial identification technique using high-quality photographs. Journal of forensic sciences, 52(4), 779-783. 
Research, Society and Development, v. 10, n. 2, e60010212469, 2021

(CC BY 4.0) | ISSN 2525-3409 | DOI: http://dx.doi.org/10.33448/rsd-v10i2.12469

Kleinhans, C. (2004). Virtual child porn: The law and the semiotics of the image. Journal of visual culture, 3(1), 17-34.

Liberati, A., Altman, D. G., Tetzlaff, J., Mulrow, C., Gøtzsche, P. C., Ioannidis, J. P. A., Clarke, M., Devereaux, P. J., Kleijnen, J., \& Moher, D. (2009). The PRISMA statement for reporting systematic reviews and meta-analyses of studies that evaluate health care interventions: Explanation and elaboration. Journal of Clinical Epidemiology, 62(10), e1-e34. https://doi.org/10.1016/j.jclinepi.2009.06.006

Lopes, J. R. (2012). Eficácia do método de estimativa de idade de Lamendin [PhD Thesis]. Universidade de São Paulo.

Machado, C. E. P., Flores, M. R. P., Lima, L. N. C., Tinoco, R. L. R., Franco, A., Bezerra, A. C. B., Evison, M. P., \& Guimaraes, M. A. (2017). A new approach for the analysis of facial growth and age estimation: Iris ratio. PloS One, 12(7), e0180330. https://doi.org/10.1371/journal.pone.0180330

Maia, L. C., \& Antonio, A. G. (2012). Systematic reviews in dental research. A guideline. Journal of Clinical Pediatric Dentistry, 37(2), 117-124.

Moreton, R., \& Morley, J. (2011). Investigation into the use of photoanthropometry in facial image comparison. Forensic science international, 212(1-3), 231237.

Pereira, A. S., Shitsuka, D. M., Parreira, F. J., \& Shitsuka, R. (2018). Metodologia da pesquisa científica (1a). Brasil.

Ratnayake, M., Obertova, Z., Dose, M., Gabriel, P., Broker, H. M., Brauckmann, M., Barkus, A., Rizgeliene, R., Tutkuviene, J., Ritz-Timme, S., Marasciuolo, L., Gibelli, D., \& Cattaneo, C. (2014). The juvenile face as a suitable age indicator in child pornography cases: A pilot study on the reliability of automated and visual estimation approaches. International Journal of Legal Medicine, 128(5), 803-808. https://doi.org/10.1007/s00414-013-0875-y

Schaaf, H., Wilbrand, J.-F., Boedeker, R.-H., \& Howaldt, H.-P. (2010). Accuracy of Photographic Assessment Compared with Standard Anthropometric Measurements in Nonsynostotic Cranial Deformities. The Cleft Palate-Craniofacial Journal, 47(5), 447-453. https://doi.org/10.1597/09-026

Schmeling, A., Grundmann, C., Fuhrmann, A., Kaatsch, H.-J., Knell, B., Ramsthaler, F., Reisinger, W., Riepert, T., Ritz-Timme, S., Rosing, F. W., Rotzscher, K., \& Geserick, G. (2008). Criteria for age estimation in living individuals. International Journal of Legal Medicine, 122(6), 457-460. https://doi.org/10.1007/s00414-008-0254-2

Wall, S., Kazahaya, K., Becker, S. S., \& Becker, D. G. (1999). Thirty-five millimeter versus digital photography: Comparison of photographic quality and clinical evaluation. Facial plastic surgery, 15(02), 101-109. 